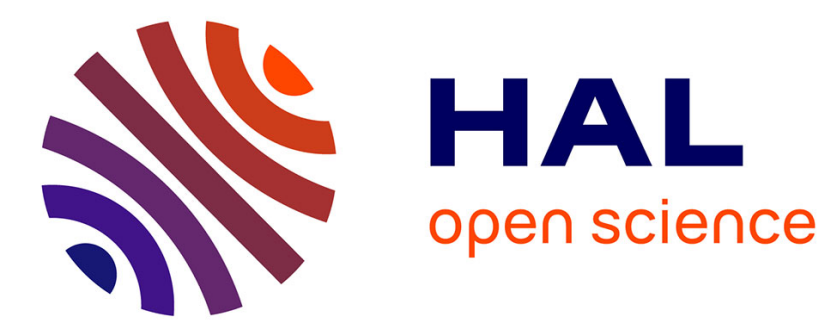

\title{
Magnétomètre à pompage optique sur les isotopes du mercure
}

\author{
G. Hanuise
}

\section{To cite this version:}

G. Hanuise. Magnétomètre à pompage optique sur les isotopes du mercure. Revue de Physique Appliquée, 1970, 5 (1), pp.119-120. 10.1051/rphysap:0197000501011900 . jpa-00243343

\section{HAL Id: jpa-00243343 https://hal.science/jpa-00243343}

Submitted on 1 Jan 1970

HAL is a multi-disciplinary open access archive for the deposit and dissemination of scientific research documents, whether they are published or not. The documents may come from teaching and research institutions in France or abroad, or from public or private research centers.
L'archive ouverte pluridisciplinaire HAL, est destinée au dépôt et à la diffusion de documents scientifiques de niveau recherche, publiés ou non, émanant des établissements d'enseignement et de recherche français ou étrangers, des laboratoires publics ou privés. 


\title{
MAGNÉTOMÈTRE A POMPAGE OPTIQUE SUR LES ISOTOPES DU MERGURE
}

\author{
Par G. HANUISE,
}

Office National d'Études et de Recherches Aérospatiales (O.N.E.R.A.), Châtillon-sous-Bagneux (France).

Résumé. - Le dispositif de résonance magnétique nucléaire utilisant un champ alternatif parallèle au champ directeur présente une sensibilité intéressante aux champs magnétiques faibles. Cette sensibilité est proportionnelle au temps de relaxation du noyau résonnant.

L'isotope $199 \mathrm{du}$ mercure a un temps de relaxation élevé, supérieur à $100 \mathrm{~s}$. Grâce aux techniques de pompage optique et de détection optique non résonnante par effet Faraday, et à l'aide d'un asservissement du champ directeur au moyen de l'isotope $201 \mathrm{du}$ mercure, il est possible de prévoir un magnétomètre ayant un seuil de détection de l'ordre de $10^{-8}$ gauss.

Abstract. - A nuclear magnetic resonance experiment with a.c. field parallel to d.c. field has a good sensitivity for weak d.c. fields. This sensitivity is proportional to the resonant nucleus relaxation time.

199 mercury has a large relaxation time, greater than $100 \mathrm{~s}$. Using optical pumping techniques, non-resonant optical detection with Faraday effect, and d.c. field control with 201 mercury, a $10^{-8}$ gauss threshold magnetometer can be designed.

Principe de l'appareil. - Il existe en résonance magnétique une configuration de champs magnétiques particulièrement favorable pour la mesure des champs magnétiques statiques faibles. Cette configuration (fig. 1) comprend un champ directeur continu $H$ et un champ alternatif $H_{1} \cos \omega t$ parallèle à $H$ et dont l'amplitude est de l'ordre de grandeur de $H$.

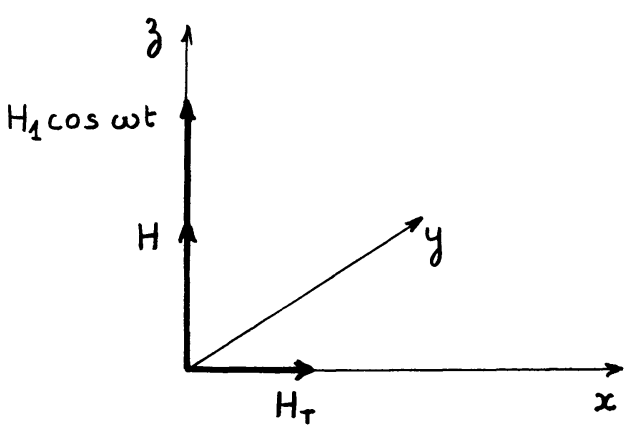

FIG. 1.

La théorie [1] montre qu'en présence d'un champ transversal $H_{T}$, il y a résonance lorsque $\omega$ et $H$ sont liés par la relation :

$$
n \omega+\gamma H=0
$$

$\gamma$ étant le rapport gyromagnétique du noyau résonnant, $n$ étant un entier positif, négatif ou nul. Si le champ $H_{T}$ est dirigé selon l'axe $O x$, on calcule les deux composantes de l'aimantation transversale dans un trièdre tournant à la vitesse angulaire :

$$
\begin{gathered}
\theta=-\left(\gamma H+\gamma H_{1} \cos \omega t\right) \\
\tilde{M}_{x}=\frac{\gamma \Delta \omega T_{2}^{2} H_{T} J_{n}\left(\gamma H_{1} / \omega\right)}{1+\left(\Delta \omega T_{2}\right)^{2}+\gamma^{2} T_{1} T_{2} H_{T}^{2} J_{n}^{2}\left(\gamma H_{1} / \omega\right)} M_{0} \\
\tilde{M}_{y}=\frac{\gamma T_{2} H_{T} J_{n}\left(\gamma H_{1} / \omega\right)}{1+\left(\Delta \omega T_{2}\right)^{2}+\gamma^{2} T_{1} T_{2} H_{T}^{2} J_{n}^{2}\left(\gamma H_{1} / \omega\right)} M_{0}
\end{gathered}
$$

avec $\Delta \omega=\gamma H+n \omega ; T_{1}, T_{2}$, temps de relaxation; $J_{n}\left(\gamma H_{1} / \omega\right)$, fonction de Bessel de première espèce d'ordre $n ; M_{0}$, aimantation de l'échantillon.

Les composantes mesurées dans le trièdre fixe sont :

$$
\begin{aligned}
& M_{x}=\tilde{M}_{x} \cos \theta-\tilde{M}_{y} \sin \theta \\
& M_{y}=\tilde{M}_{x} \sin \theta+\tilde{M}_{y} \cos \theta .
\end{aligned}
$$

La mesure de $\tilde{M}_{y}$ à la résonance permet d'obtenir la valeur de $H_{T}$.

L'analyse de ces résultats montre que, pour réaliser un magnétomètre sensible, il faut remplir les conditions suivantes :

- Obtenir un échantillon ayant un temps de relaxation long, la sensibilité étant proportionnelle à $\gamma T_{2} J_{n}\left(\gamma H_{1} / \omega\right)$;

- Se placer à la résonance $(\Delta \omega=0)$ pour avoir la sensibilité maximale.

Pour pouvoir obtenir une valeur de la fonction de Bessel maximale avec un champ alternatif $H_{1}$ techniquement réalisable, on est conduit à choisir $n$ petit, et d'autre part, à travailler à une fréquence $\omega$ faible. Le temps de relaxation très long peut être obtenu avec des gaz, sans que se pose le problème de l'inhomogénéité du champ grâce au « rétrécissement par le mouvement ». Mais la détection radio-électrique de la résonance est alors impossible.

Ces différents problèmes peuvent être résolus par l'emploi du pompage optique, dans le cas des isotopes 199 et 201 du mercure [2] :

a) Dans une cellule en quartz, le mercure 199 a des temps de relaxation supérieurs à $100 \mathrm{~s}$.

b) Le pompage optique par une lampe à mercure 204 permet d'obtenir un signal facilement détectable, bien que la pression dans la cellule soit faible (environ $10^{-3}$ torr). Le pompage optique diminue le temps de relaxation apparent du noyau résonnant, mais cet effet peut être maintenu assez faible. 
c) La détection du signal de résonance s'effectue par la méthode du faisceau croisé. L'emploi d'une lampe à mercure 202 permet une détection par effet Faraday, en lumière non résonnante, sans diminution du temps de relaxation apparent [3].

d) L'isotope 201, placé dans la même cellule, subit aussi le cycle de pompage optique dans ces conditions. Il permet la stabilisation du champ directeur $H$ par un asservissement utilisant la raie de résonance et une fréquence fixe $\omega_{s}$.

Projet de magnétomètre (fig. 2). - Le projet suivant utilise les différentes techniques présentées.

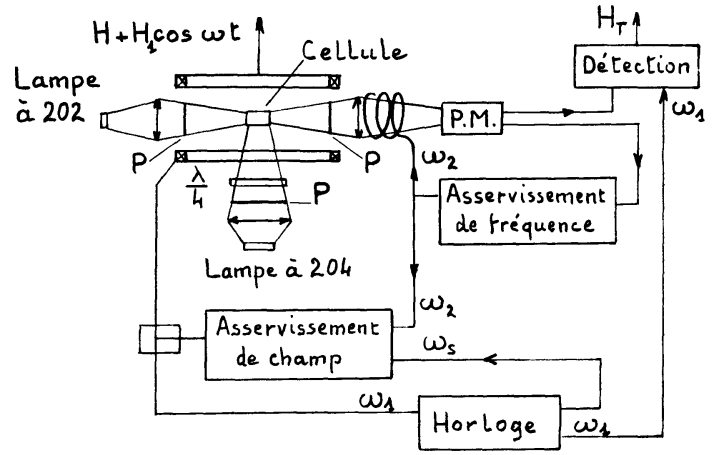

FIG. 2.
La cellule contenant les isotopes 199 et 201 est placée dans le champ $H+H_{1} \cos \omega_{1} t$, avec $\omega_{1}=\gamma_{1} H$ $\left(\gamma_{1}\right.$, rapport gyromagnétique de l'isotope 199). Une bobine fournit le champ alternatif $H_{2} \cos \omega_{2} t$ avec $\omega_{2}=\gamma_{2} H \quad\left(\gamma_{2}\right.$, rapport gyromagnétique de l'isotope 201) permettant d'asservir le champ $H$ à une valeur fixe.

Deux systèmes optiques permettent :

- l'un le pompage le long de $H$ par emploi de lumière polarisée circulairement émise par une lampe contenant l'isotope 204,

- l'autre la détection des signaux perpendiculairement à $H$ par de la lumière polarisée linéairement, émise par une lampe à isotope 202.

Les signaux de résonance sont recueillis par un photomultiplicateur. Un asservissement de champ permet de maintenir $H$ à la valeur $\omega_{s} / \gamma_{2}$, la fréquence $\omega_{s}$ étant fournie par une horloge qui fournit aussi $\omega_{1}$.

Le signal à la fréquence $\omega_{1}$, ou à l'un de ses harmoniques, a une amplitude fournissant la valeur de $H_{T}$. Une détection appropriée sépare les deux composantes de $H_{T}$ perpendiculaires à $H$.

En utilisant les valeurs déjà obtenues des différents paramètres, on peut estimer le seuil de détection à $10^{-8} \mathrm{Oe}$ environ.

\section{BIBLIOGRAPHIE}

[1] Hanuise (G.), Étude théorique et expérimentale du gyromètre à induction nucléaire. Publication O.N.E.R.A. no 118, 1967, Thèse 1967.

[2] CAGNAC (B.), Orientation nucléaire par pompage optique des isotopes du mercure, Annales de Physique, 1961, 6, 467.

[3] Cohen-TAnnoudji (C.), Théorie quantique du cycle de pompage optique, Annales de Physique, 1962, 7, 423-469. 